\title{
Open-field behavior in mice: Effect of test illumination'
}

W. E. McREYNOLDS, MORTON W. WEIR AND J. C. DEFRIES ${ }^{2}$

UNIVERSITY OF ILLINOIS

The open-field behavior of two inbred strains of mice $(B A L B / c J$ and $C 57 B L / 6 J)$ was observed under five levels of test illumination. Increased level of test illumination resulted in reduced activity in both strains, with the albino $B A L B$ strain more greatly affected than the pigmented C57BL strain. In addition, open-field defecation scores of the BALB strain were increased as a function of increasing test illumination, whereas those of the C57BL strain were little affected. These results support the hypothesis that differences in open-field behavior are due in part to a visually mediated fear reaction which is a function of test illumination.

C57BL/6J mice (a pigmented strain) have higher activity and lower defecation scores than BALB/cJ mice (albino) when tested in the open field under conditions of moderately bright illumination (approximately $40 \mathrm{ft}-\mathrm{c}$ ). In segregating $\mathrm{F}_{2}, \mathrm{~F}_{3}$, and $\mathrm{F}_{4}$ generations, pigmented animals have also been found to have relatively higher activity and lower defecation scores than albinos, indicating that approximately $20 \%$ of the difference between the original inbred strains in both open-field activity and defecation was due to a single-gene difference at the albino locus (DeFries et al, 1966). These differences persisted when animals of an $\mathrm{F}_{5}$ generation were tested under white light, but largely disappeared when tested under red light; therefore, it was suggested that albino mice are more photophobic than pigmented animals.

If differences in open-field behavior are due in part to a visually mediated fear reaction which is a function of illumination, then members of both inbred strains should manifest increased "emotionality" (higher defecation and lower activity) as level of test illumination is increased. However, if albinos are more photophobic than pigmented animals, members of the BALB/cJ strain should be more affected than those of the C57BL/6J strain by changes in test illumination, at least at the lower levels. In order to investigate these possibilities, the open-field behavior of these two inbred strains of mice was observed under five levels of illumination ( 0 to $480 \mathrm{ft}-\mathrm{c}$ ) in approximately equal $\log$ steps.

\section{Method}

Members of two inbred strains of mice (BALB/cJ and $\mathrm{C57BL} / 6 \mathrm{~J}$ ) were mated within strains at $70 \pm 10$ days of age and the resulting inbred offspring were tested at $40 \pm 2$ days of age in a square ( $24 \times 24 \times$ 8 in.) open field. The floor and sides of the field were of white, painted Plexiglas. Two sets of five light sources were beamed through holes and red filters to photoconductive cells on the opposite side, effectively dividing the floor into 36 squares (4 $\mathrm{x}$ 4 in. each), and the number of light beams broken was automatically recorded on counters. A GE floodlight (QF-500A) mounted $48 \mathrm{in}$, above the center of the floor of the field provided illumination during testing. Level of illumination was controlled by using various combinations of brass, wire-mesh filters (20 to 100 holes per sq in.) mounted at the base of the light source. ${ }^{3}$ A 5 in. squirrel-cage blower circulated air between the lamp and the wire-mesh filters. The lowest level of illumination was darkness, whereas the floodlight with no filter provided the highest level (480 apparent $\mathrm{ft}-\mathrm{c})$. The three intermediate levels $(4.5,18.9$, and 98.9 apparent $\mathrm{ft}-\mathrm{c})$ were selected so as to provide approximately equal log steps between the lowest and highest levels. All levels of illumination were measured with a MacBeth Illuminometer.

Each mouse was tested for $3 \mathrm{~min}$ on each of two successive days between 1:00 and 8:00 PM, i.e., during the latter part of the light phase of a $12 \mathrm{~h}$ light-dark cycle. The total number of light beams interrupted and the total number of fecal boluses dropped during the two test periods were used as activity and defecation scores. Both activity and defecation scores were subjected to square root transformations; since defecation scores were low, 0.5 was added to these raw scores prior to transformation. Sixteen Ss of each sex and each strain were tested in the open field under each of the five levels of illumination, resulting in a 2 by 2 by 5 factorial arrangement of treatments ( 2 strains, 2 sexes, and 5 levels of illumination) with a total of $320 \mathrm{Ss}$.

\section{Results and Discussion}

The mean transformed open-field activity and defecation scores for members of the BALB/CJ and C57BL/ $6 \mathrm{~J}$ strains, expressed as a function of level of test illumination, are summarized in Figs. 1 and 2. With regard to activity, main effects due to strain and level of illumination were highly significant ( $F=326.3$, $\mathrm{df}=1 / 300, p<.001 ; F=62.0, \mathrm{df}=4 / 300, p<.001)$. The mean transformed activity scores of the C57BL and BALB strains were 17.4 and 10.7 , respectively, accounting for the highly significant main effect due to the difference between strains. As may be seen in Fig. 1, the level of activity in both strains decreases as level of test illumination increases, resulting in the significant main effect due to illumination. Orthogonal coefficients were used to partition this main 


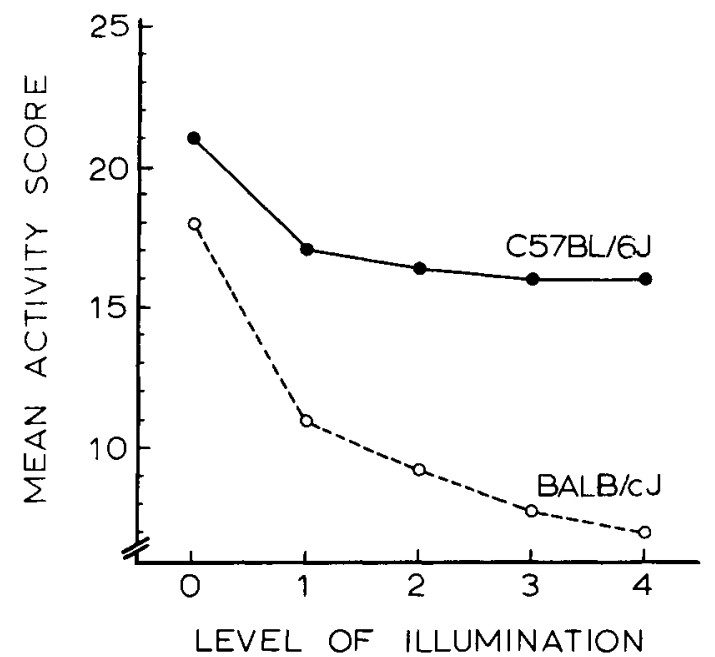

Fig. 1. Mean transformed open-field activity scores of $B A L B / c J$ and C57BL/6J mice as a function of level of test illumination (0 to 480 apparent ft-c, in approximately equal log steps).

effect into its linear, quadratic, cublc and quartic components (1inear, $\mathrm{F}=192.2, \mathrm{p}<.001$; quadratic, $\mathrm{F}=$ $46.3, p<.001$; cubic, $F=7.69, p<.01$; quartic, $F=1.73$, $p>.05$; all with $d f=1 / 300$ ). The only other significant effect was associated with the interaction between strain and level of test illumination $(F=8.1$, df $=$ $4 / 300, p<.001$ ) and only the linear component contributed significantly to this interaction $(F=29.1, \mathrm{df}=$ $1 / 300, p<.001)$.

The mean transformed defecation scores of the C57BL and BALB strains were 1.3 and 2.6, respectively, resulting in a highly significant main effect due to the difference between strains $(F=444.3, \mathrm{df}=$ $1 / 300, p<.001)$. The interaction between strain and level of test illumination approached significance $(F=$ 2.1, $\mathrm{df}=4 / 300, .05<\mathrm{p}<.10$ ) and the linear component again contributed significantly to this interaction $(F=$ $6.7, \mathrm{df}=1 / 300, \mathrm{p}<.02$ ). Males had somewhat higher defecation scores than females $(2.0$ vs 1.8$)$, resulting in the only other significant effect $(F=5.1, \mathrm{df}=$ $1 / 300, p<.05$ ).

The results of the present study indicate that the open-field behavior of the BALB/CJ and C57BL/6J strains of mice is differentially influenced by level of test illumination. Conflicting results regarding the effect of test lllumination on activity level in different inbred strains of mice have been reported previously (McClearn, 1960; Ross et al, 1966; Brookshire \& Rieser, 1967). However, different strains and substrains of mice and different test conditions were employed in these studies and the levels of test illumination were relatively low.

Although both strains used in this study manifested reduced open-field activity as a function of increased level of illumination, the BALB (albino) strain was

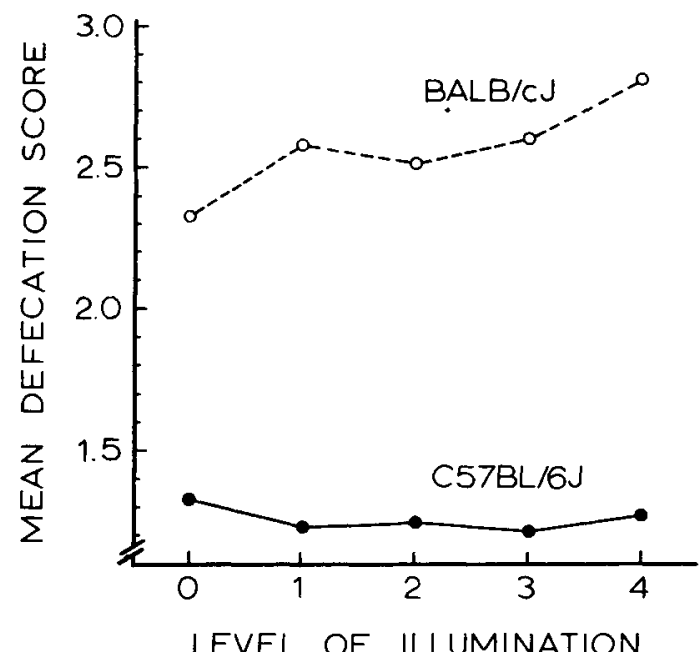

Fig. 2. Mean transformed open-field defecation scores of BALB/cJ

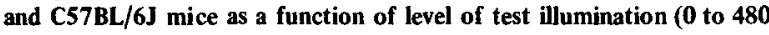
apparent $\mathrm{ft}-\mathrm{c}$, in approximately equal log steps).

more greatly affected than the pigmented C57BL strain. In addition, increasing level of test illumination was accompanied by a corresponding increase in openfield defecation in the BALB strain, whereas defecation scores of the C57BL strain were little affected. It should be noted that these two inbred strains possess many differences in addition to that of eye pigmentation. For example, differences in activity and defecation at the 0-level of illumination must be due to differences between the strains which are independent of visual system differences. Nevertheless, the fact that both strains are affected by increasing level of test illumination indicates that open-field behavior under conditions of moderate or bright illumination is at least in part a function of a visually mediated fear reaction. The differential effect of increasing illumination upon the open-field behavior of these two strains also indicates that albinos are more photophobic than pigmented animals.

\section{References}

BROOKSHIRE, K. H., \& RIESER, T. C. Temporal course of exploratory activity in three inbred strains of mice. J. comp. physiol. Psychol, $1967,63,549-551$.

DeFRIES, J. C., HEGMANN, J. P., \& WEIR, M. W. Open-field behavior in mice: Evidence for a major gene effect mediated by the visual system. Science, 1966, 154, 1577-1579.

McCLEARN, G. E. Strain differences in activity of mice: Influence of illumination. J. comp. physiol Psychol, 1960, 53, 142-143.

ROSS, S., NAGY, Z. M., KESSLER, C., \& SCOTT, J. P. Effects of illumination on wall-leaving behavior and activity in three inbred mouse strains. J. comp. physiol. Psychol, 1966, 62, 338-340.

Note

1. Supported in part by NIH grant GM-12486.

2. Present address: Institute for Behavioral Genetics, University of Colorado, Boulder.

3. We thank Dr. Jozef Cohen for suggesting this method of controlling level of illumination. 